\title{
Outcome of ultrasound guided foam sclerotherapy (UGFS) treatment for varicose veins: A four years retrospective study at a tertiary care hospital in north India
}

\author{
Singh $\mathbf{S}^{1}$, Maurya A.K. ${ }^{2}$, Singh $\mathbf{M}^{3}$, Sachdeva $\mathrm{V}^{4}$ \\ ${ }^{1}$ Dr Sanjay Singh, General Surgery, General Surgeon, ${ }^{2}$ Dr Akhilesh Kumar Maurya, Senior Resident in Department of \\ Surgery, MLN Medical, College, Allahabad, India, ${ }^{3}$ Dr Mayurika Singh, Radiodiagnosis, Radiologist, ${ }^{4}$ Dr Vikas \\ Sachdeva, PG Department of Surgery SRN Hospital \& MLN Medical College, Allahabad, Uttar Pradesh, India.
}

Address for Correspondence: Dr Akhilesh Kumar Maurya, Email: dr.akhilbrd@gmail.com

\begin{abstract}
Objective:The purpose of this study was to determine the outcome and rate of recurrence for varicose veins treated with ultrasound-guided foam sclerotherapy (UGFS). Methods: Data was collected from hospital records. Patients treated with UGFS for superficial venous insufficiency were included in the study. Total 361 legs in 241 patients, out of which 121 patients with unilateral limbs and 120 patients with bilateral limbs were found to be treated by this method. Results: Out of these 361 legs great saphenous vein (GSV) varicosity was found in 88 legs, short saphenous vein (SSV) in 76, GSV \& SSV both in 63, others 134. 264 legs with primary varicosity while 97 were with recurrent. In 294 legs clinical CEAP was 2-3 while in 67 legs it was 4-6. Saphenofemoral junction (SFJ) incompetence was found 60 legs, saphenopopliteal junction (SPJ) incompetence in 68, only perforator's incompetence was in 56, SFJ with perforator's incompetence 61, SPJ with perforator's 29 and no incompetence was noted in 87 legs.Outcome at 6 months was $96.23 \%$ while treatment failure and recurrences were noted in $2.77 \%$ of legs. Complications which were noted at 1 week were superficial skin necrosis in $3.04 \%$, pain at injection sites in $15.23 \%$, superficial thrombophlebitis in $16.62 \%$, bruising in $12.18 \%$, skin staining in $11.08 \%$, superficial vein thrombosis (SVT) in $9.97 \%$ while no DVT was noted in any of the treated legs. Conclusion: On conclusion it was found that UGFS is a popular office based treatment modality, safe, effective, easy and improvement in venous signs and symptoms. Even on recurrence patients easily accepts retreatment with this method. Furthermore it is associated with lesser pain, anesthesia requirements; time off work and driving gives it additional advantages.
\end{abstract}

Keywords: Foam Sclerotherapy, Superficial Venous Insufficiency, Ultrasound Guided Sclerotherapy, UGFS Outcome, Varicose veins.

\section{Introduction}

There are two venous systems in the leg: First deep venous system; and second superficial venous system. When superficial veins under the skin become dilated, bulging and twisted then they are known as varicose veins. Ultrasound guided foam sclerotherapy (UGFS) is a well-established method for treatment of superficial venous insufficiency. First technique of foam preparation and injection was described in 1944 by Orbach[1]. The benefit of foam over liquid is that it displaces the blood and fill the vein; the foam is not

Manuscript received $26^{\text {th }}$ March 2016

Reviewed: $9^{\text {th }}$ April 2016

Author Corrected: $18^{\text {th }}$ April 2016

Accepted for Publication $30^{\text {th }}$ April 2016 diluted by blood; very small amount is needed to obtain the same effect. Once the foam reaches large veins and mixes with blood it is inactivated because blood is a strong inhibitor of sclerosants. Air from the foam is rapidly absorbed from the vein left the sclerosant for the action. Use of ultrasound guidance offers additional benefits over blind procedure is that the foam can be seen during injection and can be manipulated once injected into the veins. Without ultrasound guidance chances and quantity of foam to reach deep venous system is increased so the chances of deep vein thrombosis (DVT) are increased. Reasons behind popularity of this method are its easy availability, 
simplicity, no requirement of analgesics and anesthesia, office based procedure, low cost, patient satisfaction, lesser complications and comparable efficacy with other methods[3,5]. Other common modalities which are being used for treatment of varicose veins are saphenous vein striping (SVS), radiofrequency ablation (RFA) and endovenous laser ablation (EVLA). Requirement of anesthesia, operation theatre (OT) setup and cost of treatment are the major disadvantages of these methods[3,5].

\section{Material \& Methods}

This study was carried out in Swaroop Rani Nehru (SRN) Hospital associated Motilal Nehru (MLN) Medical College, Allahabad, India after taking approval from ethics committee. Records of the patients treated by this modality were taken since January 2012 to February 2016. Inclusion criteria was patients aged above 18 years, clinical CEAP (clinical, etiological, anatomical, pathological elements) score above 4 or clinical CEAP score 2-4, having truncal varicose veins, great saphenous vein (GSV) (FIG. 1), small saphenous vein (SSV) (FIG. 2), anterior accessory saphenous vein (AASV) and other recurrent veins (FIG. 4), with or without incompetent deep venous connection, bleeding varicose veins, varicose ulcers and with skin complications. Exclusion criteria were patients below 18 years of age. Follow up period was 1 year.

Technique:The UGFS procedure have been described in detail elsewhere[3]. In brief UGFS consists of 4 steps: cannulation of veins; preparation of foam by Tessari's technique[2]; injection of prepared foam into the veins; and compression bandaging.

Outcome measures: Complete occlusion of the saphenous trunk or disappearance of other varicosities from the level of incompetence between superficial and deep venous system was taken as successful treatment. If complete occlusion was not achieved or residual varicosity was noticed at 1 week then it was considered as short term treatment failure and procedure was repeated.

\section{Results}

Out of these 361 legs great saphenous vein (GSV) varicosity was found in 88 legs, short saphenous vein (SSV) in 76, GSV \& SSV both in 63, others 134. 264 legs with primary varicosity while 97 were with recurrent. In 294 legs clinical CEAP was 2-3 while in 67 legs it was 4-6 (table 1). Saphenofemoral junction (SFJ) incompetence was found 60 legs, saphenopopliteal junction (SPJ) incompetence in 68, only perforator's incompetence was in 56, SFJ with perforator's incompetence 61, SPJ with perforator's 29 and no incompetence was noted in 87 legs (table 2). Total 241 patients were included in the study; 181 males and 60 females. 120 patients were having bilateral limbs and 121 with unilateral disease (table 3).

Table 1: Clinical details of total 361 limbs.

\begin{tabular}{|l|l|l|l|l|l|l|l|l|}
\hline Varicosities & $\begin{array}{l}\text { GSV } \\
\text { only }\end{array}$ & $\begin{array}{l}\text { SSV } \\
\text { only }\end{array}$ & $\begin{array}{l}\text { GSV } \\
\text { \& } \\
\text { SSV }\end{array}$ & Others & Primary & Recurrent & $\begin{array}{l}\text { Uncomplicated } \\
\text { (CEAP 2-3) }\end{array}$ & $\begin{array}{l}\text { Complicated } \\
\text { (CEAP 4-6) }\end{array}$ \\
\hline N & 88 & 76 & 63 & 134 & 264 & 97 & 294 & 67 \\
\hline
\end{tabular}

GSV, Great saphenous vein; SSV, Small saphenous vein; CEAP, Clinical, Etiological, Anatomical, Pathological.

Table 2: Duplex findings in 361 limbs.

\begin{tabular}{|l|l|l|l|l|l|l|}
\hline Incompetence & SFJ only & SPJ only & $\begin{array}{l}\text { Perforator's } \\
\text { only }\end{array}$ & $\begin{array}{l}\text { SFJ \& } \\
\text { Perforator's }\end{array}$ & $\begin{array}{l}\text { SPJ \& } \\
\text { Perforator's }\end{array}$ & None \\
\hline N & 60 & 68 & 56 & 61 & 29 & 87 \\
\hline
\end{tabular}

SFJ, Saphenofemoral junction; SPJ, Saphenopopliteal junction

Table 3: Patients demography.

\begin{tabular}{|l|l|l|l|l|l|l|}
\hline Variables & $\begin{array}{l}\text { Total } \\
\text { patients }\end{array}$ & $\begin{array}{l}\text { Male } \\
\text { patients }\end{array}$ & $\begin{array}{l}\text { Female } \\
\text { patients }\end{array}$ & Total limbs & $\begin{array}{l}\text { Patients with } \\
\text { Bilateral } \\
\text { limbs }\end{array}$ & $\begin{array}{l}\text { Patients with } \\
\text { Unilateral } \\
\text { limbs }\end{array}$ \\
\hline $\mathrm{N}$ & 241 & 181 & 60 & 361 & 120 & 121 \\
\hline
\end{tabular}


Table 4: Immediate complications in 361 treated limbs.

\begin{tabular}{|l|l|}
\hline Complications & $\mathbf{N}(\mathbf{\%})$ \\
\hline Presyncope before injection & $4(1.1)$ \\
\hline Presyncope post injection & $7(1.9)$ \\
\hline Extravasations & $15(4.1)$ \\
\hline Pain in leg/at the site of injection & $12(3.3)$ \\
\hline Minor bleeding & $5(1.3)$ \\
\hline Visual scotoma & $1(0.2)$ \\
\hline Severe headache & $1(0.2)$ \\
\hline Eye pain & $1(0.2)$ \\
\hline
\end{tabular}

Table 5: Early complications in 361 treated limbs.

\begin{tabular}{|l|l|}
\hline Complications & $\mathrm{N}(\%)$ \\
\hline Superficial skin necrosis & $11(3.0)$ \\
\hline Pain at injection sites & $55(15.2)$ \\
\hline Superficial thrombophlebitis & $60(16.6)$ \\
\hline Bruising & $44(12.2)$ \\
\hline Skin staining & $40(11.1)$ \\
\hline Deep vein thrombosis (DVT) & 0 \\
\hline Superficial vein thrombosis (SVT) & $36(10.0)$ \\
\hline Treatment failures \& recurrences & $10(2.8)$ \\
\hline
\end{tabular}

Table 6: Anaphylactic reactions with UGFS.

\begin{tabular}{|l|l|l|l|l|l|}
\hline Studies & $\begin{array}{l}\text { Bradbury et }_{\text {al. }^{[10]}} \\
\mathrm{N}\end{array}$ Scurr et al. $^{[18]}$ & Brzoza et al. $^{[19]}$ & Guex et al. $^{[20]}$ & Jia X et al. $^{[11]}$ \\
\hline
\end{tabular}

UGFS, Ultrasound Guided Foam Sclerotherapy

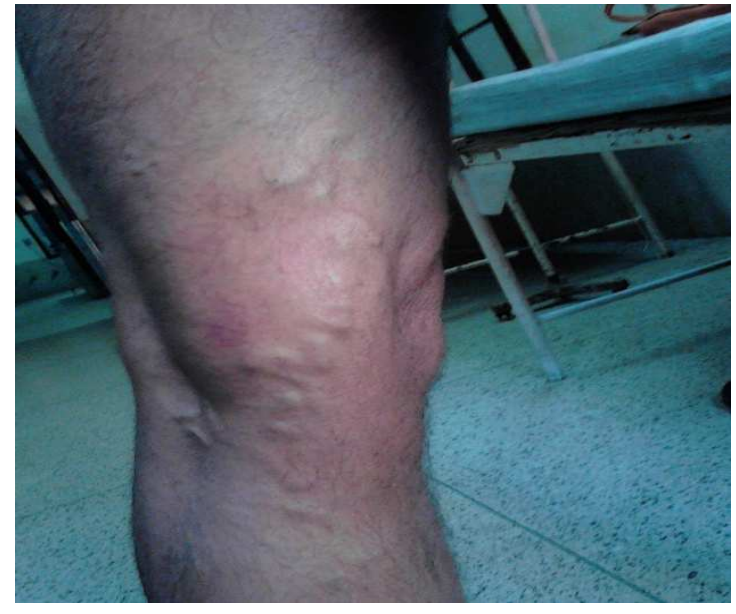

Fig 1: Great saphenous vein varicosity

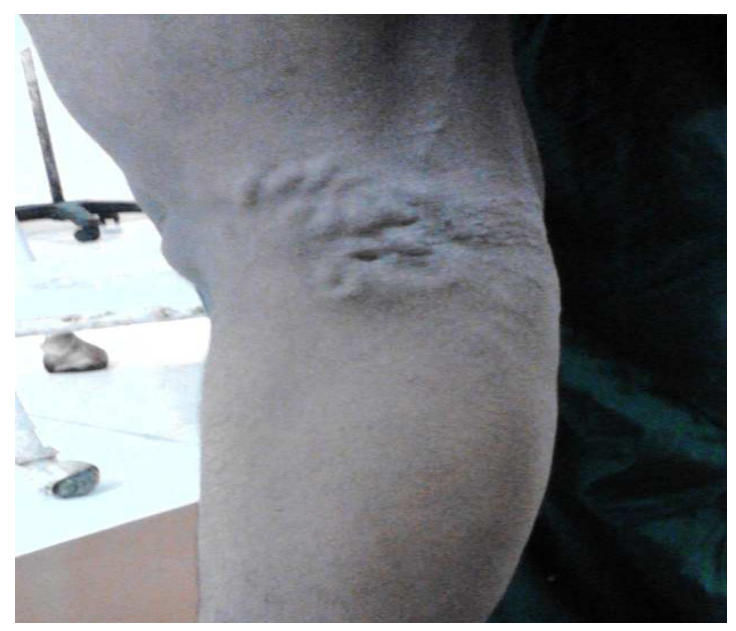

Fig 2: Small saphenous vein varicosity

Outcome at 6 months was $96.2 \%$ while treatment failure and recurrences were noted in $2.8 \%$ of legs. Immediate adverse effects were self-limiting and shown in table 4 . Complications which were noted at 1 week were superficial skin necrosis (FIG. 3) in 3.0\%, pain at injection sites in $15.2 \%$, superficial thrombophlebitis in $16.6 \%$, bruising in $12.2 \%$, skin staining in $11.1 \%$, superficial vein thrombosis (SVT) in $10.0 \%$ while no DVT was noted in any of the treated legs (table 5). All of these complications resolved without any treatment. 


\section{Discussion}

This article shows the experience of our centre in treating 361 limbs with varicose veins by this method. Success rate was $97.2 \%$ at a median follow up period of one year. Many papers have described particular problems in the management of varicose veins out of which neovascularization is a common problem and leading cause of poor outcome in surgically treated patients for recurrent varices[4].

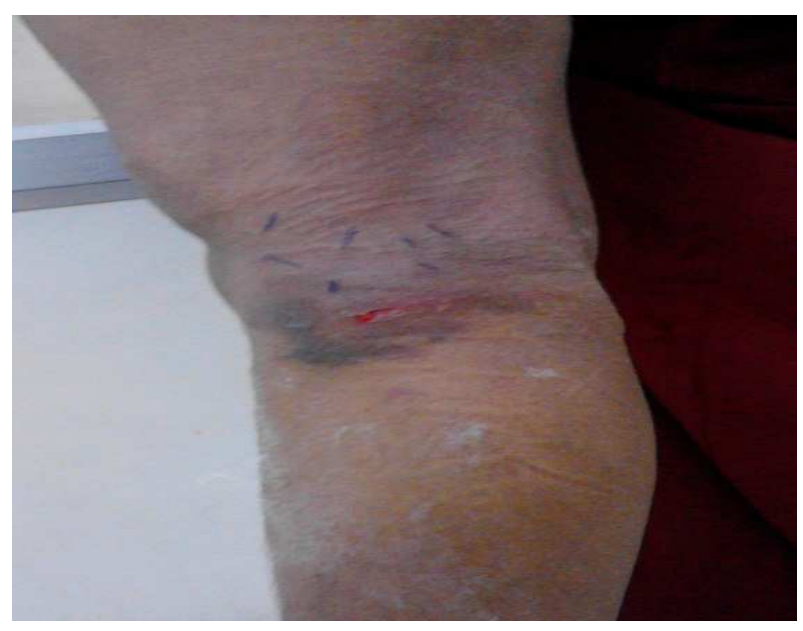

Fig 3: Superficial skin necrosis after treatment

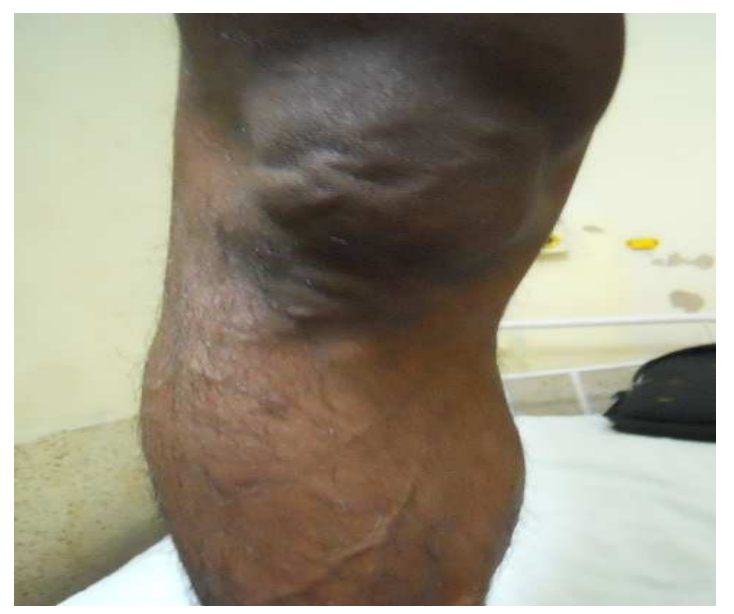

Fig 4: Recurrent varicose vein after endovenous laser ablation

Table 7: Incidence of different complications with UGFS in different studies

\begin{tabular}{|c|c|c|c|c|c|c|c|c|}
\hline Studies & $\begin{array}{l}\text { Superfici } \\
\text { al skin } \\
\text { necrosis }\end{array}$ & $\begin{array}{l}\text { Pain at } \\
\text { injectio } \\
\text { n sites }\end{array}$ & $\begin{array}{l}\text { Superficial } \\
\text { thrombophleb } \\
\text { itis }\end{array}$ & $\begin{array}{l}\text { Bruising\& } \\
\text { pigmentat } \\
\text { ion }\end{array}$ & $\begin{array}{l}\text { Skin } \\
\text { staining }\end{array}$ & DVT & SVT & $\begin{array}{l}\text { Treatment } \\
\text { failures \& } \\
\text { recurrences }\end{array}$ \\
\hline Present study & 3.0 & 15.2 & 16.6 & 12.2 & 11.1 & 0 & 10.0 & 2.8 \\
\hline $\begin{array}{l}\text { Chapman smith P et. } \\
\mathrm{Al}[6]\end{array}$ & - & 3 & 10.3 & - & 3.9 & 1 & - & $23-43$ \\
\hline Jia X et. Al[11] & $0-1.3$ & $0.3-4.2$ & $0.05-9.2$ & $19.8-31.6$ & $7.8-55.1$ & $\begin{array}{l}0.02- \\
0.7\end{array}$ & $\begin{array}{l}0.1- \\
8.8\end{array}$ & $0.5-5.9$ \\
\hline Brunken A et. Al[7] & - & - & 14.9 & - & - & 0 & - & 20 \\
\hline Blaise $S$ et. $\mathrm{Al}[22]$ & - & - & - & $6-9$ & - & - & - & - \\
\hline $\begin{array}{l}\text { Figueiredo } M \text { et. } \\
\text { Al[17] }\end{array}$ & - & - & 37.1 & 28.6 & - & 14.3 & - & 54.2 \\
\hline $\begin{array}{l}\text { Evi Kalodiki et. } \\
\text { Al[27] }\end{array}$ & - & - & 7.7 & 15 & 1.5 & - & - & - \\
\hline Shadid N et. Al[28] & - & 2.6 & 7.4 & 0 & 5.6 & 0.4 & - & 19.3 \\
\hline $\begin{array}{l}\text { Bradbury AW et. } \\
\text { Al[10] }\end{array}$ & - & 0.24 & - & - & - & 0.24 & - & 12.9 \\
\hline Myers KA et. Al[29] & - & - & - & - & - & - & - & $47.6-23.2$ \\
\hline Gillet JL et. Al[30] & - & - & - & - & - & .98 & & 9.7 \\
\hline Hamahata A et.al[31] & - & - & - & - & - & - & - & 34 \\
\hline Myers KA et. Al[32] & - & - & - & - & - & 1.45 & - & - \\
\hline $\begin{array}{l}\text { Hamel-Desnos C et. } \\
\text { Al[33] }\end{array}$ & - & - & - & - & - & - & - & 23.2 \\
\hline $\begin{array}{l}\text { Maurya AK et. } \\
\text { Al[34] }\end{array}$ & 2.7 & 14.18 & 14.18 & 12.16 & 8.78 & 0 & 6.75 & 0 \\
\hline
\end{tabular}

UGFS, Ultrasound Guided Foam Sclerotherapy; DVT, Deep Vein Thrombosis; SVT, Superficial Vein Thrombosis 
Most common immediate complication noticed in this study was extravasation of foam during injection (4.1\%), which is similar to other studies[5]. Superficial thrombophlebitis incidence in this study was $16.6 \%$ which was similar to different studies[6,7,8] except one who shows its incidence 39\%[9]. Other adverse effects in this study were superficial skin necrosis, pain at injection sites, bruising \& pigmentation, skin staining and SVT. The incidences of these adverse events in different studies have been described in table 7 and table 8.Rasmussen LH et. Al[16] reported one case of DVT with SVS in their RCT. Most of these complications were found to be self-limiting and minor so UGFS can be considered as a safe procedure.Systemic complications of foam sclerotherapy like Photopsia, Transient blurring of vision, Transient ischemic attack, Headache, Chest tightness \& dry cough seen with both liquid and foam sclerotherapy has been reported in less than $1 \%$ of the patients[6,10,11,12]. A study came with comparison of carbon dioxide foam with air foam. Use of $\mathrm{CO} 2$ foam was associated with substantial reduction these systemic complications[21].

Table 8: Incidence of different complications with SVS, RFA and EVLA in different studies.

\begin{tabular}{|c|c|c|c|c|c|c|c|c|}
\hline \multirow[t]{2}{*}{ Complications } & \multicolumn{4}{|l|}{ SVS } & \multicolumn{2}{|l|}{ RFA } & \multicolumn{2}{|l|}{ EVLA } \\
\hline & $\begin{array}{l}\text { Lurie } \\
\mathrm{F} \text { et. } \\
\mathrm{Al}^{[23]}\end{array}$ & $\begin{array}{l}\text { Subramonia } \\
\mathrm{S} \text { et. } \mathrm{Al}^{[24]}\end{array}$ & $\begin{array}{l}\text { Evi } \\
\text { Kalodiki } \\
\text { et. } \mathrm{Al}^{[27]}\end{array}$ & $\begin{array}{l}\text { Shadid } \\
\mathrm{N} \text { et. } \\
\mathrm{Al}^{[28]}\end{array}$ & $\begin{array}{l}\text { Lurie } \mathrm{F} \\
\text { et. } \mathrm{Al}^{[23]}\end{array}$ & $\begin{array}{l}\text { Vasquez } \\
\text { MA et. } \\
\mathrm{Al}^{[25]}\end{array}$ & $\begin{array}{l}\text { Rasmussen } \\
\mathrm{LH} \quad \text { et. } \\
\mathrm{Al}^{[16]}\end{array}$ & $\begin{array}{l}\text { Christenson } \\
\text { JT et. } \mathrm{Al}^{[26]}\end{array}$ \\
\hline $\begin{array}{ll}\text { Bruising } & \& \\
\text { pigmentation } & \end{array}$ & 0 & 93.54 & 4.6 & 1.1 & 0 & 0.6 & 0 & 15 \\
\hline DVT & 0 & - & - & 0 & 0 & 0.14 & 0 & 0 \\
\hline $\begin{array}{l}\text { Superficial } \\
\text { thrombophlebitis }\end{array}$ & - & - & 0 & 0 & 0 & 12 & 0 & 4 \\
\hline $\begin{array}{l}\text { Pain at injection } \\
\text { sites/leg }\end{array}$ & 25.0 & - & - & 0 & 4.5 & - & 2.58 & - \\
\hline Paraesthesia & 5.6 & 8 & - & 3 & 11.4 & 0.3 & 0 & 1 \\
\hline Groin infection & 5.6 & - & 2 & 2 & 0 & 0.5 & 0 & 0 \\
\hline Hematoma & 38.9 & - & - & 1 & 15.9 & - & 0 & 5 \\
\hline
\end{tabular}

SVS, Saphenous Vein Striping; RFA, Radiofrequency Ablation; EVLA, Endovenous Laser Ablation; DVT, Deep Vein Thrombosis

Recurrences associated with treatment of varicose veins is a common problem and its rate varies from $4.9 \%$ to $40 \%$ in different studies $[6,13,14,15,16]$. One study shows $57 \%[17]$ failure rate while another study shows only $2 \%[6]$. Our study noted $2.8 \%$ failure rate and recurrence. Neovascularization should be kept in mind for these failures and recurrences.

Anaphylactic reactions mostly seen with liquid sclerotherapy but can be seen with foam also. Table 6 shows incidence of this complication in different reports. No case of anaphylactic reaction seen in this study.

\section{Conclusion}

On conclusion it was found that UGFS is a popular office based treatment modality, safe, effective, easy and improvement in venous signs and symptoms. Even on recurrence patients easily accepts retreatment with this method. Furthermore it is associated with lesser pain, anesthesia requirements, time off work and driving gives it additional advantages.

\section{Financial support and sponsorship}

None

\section{Conflicts of interest}

Authors hereby declare that there are no conflicts of interest in present study.
Funding: Nil, Conflict of interest: None initiated. Permission from IRB: Yes

\section{References}

1. Wollmann JC.The history of sclerosing foams. Dermatol Surg.2004May;30(5):694-703; discussion 703.

2. Tessari L, Cavezzi A, Frullini A. Preliminary experience with a new sclerosing foam in the treatment of varicose veins. Dermatol Surg. 001Jan;27(1) : 58-60.

3. Coleridge Smith P. Foam and liquid sclerotherapy for varicose veins. Phlebology. 2009;24 Suppl 1:62-72. 
doi: 10.1258/phleb.2009.09s007.

4. Winterborn RJ, Foy C, Heather BP, Earnshaw JJ. Randomised trial of flush saphenofemoral ligation for primary great saphenous varicose veins. Eur J Vasc Endovasc $\quad$ Surg. $2008 \quad$ Oct;36(4):477-84.doi: 10.1016/j.ejvs.2008.06.022. Epub 2008 Aug 20.

5. Abbassi-Ghadi N, Hafez H. Ultrasound-guided foam sclerotherapy within a rolling treatment programme is an effective low-cost treatment for superficial venous insufficiency. Phlebology. 2013 Jun;28(4):195-200. doi: 10.1258/phleb.2011.011099. Epub 2013 May 6.

6. Chapman-Smith P, Browne A. Prospective five-year study of ultrasound-guided foam sclerotherapy in the treatment of great saphenous vein reflux. Phlebology. 2009 Aug;24(4):183-8.doi:10.1258/phleb.2009. 008080.

7. Brunken A, Rabe E, Pannier F. Changes in venous function after foam sclerotherapy of varicose veins. Phlebology.2009Aug;24(4):145-50.doi:10.1258/phleb. 2009.008068.

8. Islamoglu F. An alternative treatment for varicose veins: ligation plus foam sclerotherapy. Dermatol Surg. 2011 Apr;37(4):470-9. doi: 10.1111/j.1524-4725.2011. 01927.x.

9. M Figueiredo, S Araújo, N Barros Jr, F Miranda Jr. Results of surgical treatment compared with ultrasoundguided foam sclerotherapy in patients with varicose veins: a prospective randomized study. Eur J Vasc Endovasc Surg. 2009 Dec;38(6):758-63. doi: 10.1016 /j.ejvs.2009.07.015. Epub 2009 Sep 9.

10. Bradbury AW, Bate G, Pang K, Darvall KA, Adam DJ. Ultrasound-guided foam sclerotherapy is a safe and clinically effective treatment for superficial venous reflux. J Vasc Surg. 2010 Oct;52(4):939-45. doi: 10.1016/j.jvs.2010.04.077. Epub 2010 Jul 17.

11. Jia X, Mowatt G, Burr JM, Cassar K, Cook J, Fraser C. Systematic review of foam sclerotherapy for varicose veins. Br J Surg. 2007 Aug;94(8):925-36.

12. Guex JJ, Allaert FA, Gillet JL, Chleir F. Immediate and midterm complications of sclerotherapy: report of a prospective multicenter registry of 12,173 sclerotherapy sessions. Dermatol Surg. 2005 Feb;31(2):123-8; discussion 128.
13. Hahn M, Schulz T, Junger M. Outcome four years aftertranscatheter foam sclerotherapy of the greater saphenous vein. Phlebologie 2008;37:237-40.

14. Leopardi D, Hoggan BL, Fitridge RA, Woodruff PW, Maddern GJ. Systematic review of treatments for varicose veins. Ann Vasc Surg. 2009 Mar;23(2):264-76. doi: 10.1016/j.avsg.2008.10.007. Epub 2008 Dec 6.

15. Pang KH, Bate GR, Darvall KA, Adam DJ, Bradbury AW.Healing and recurrence rates following ultrasound-guided foam sclerotherapy of superficial venous reflux in patientswith chronic venous ulceration. Eur J Vasc Endovasc Surg 2010;40:790-5.

16. Rasmussen LH, Lawaetz M, Bjoern L, Vennits B, Blemings A,Eklof B. Randomized clinical trial comparing endovenous laser ablation, radiofrequency ablation, foam sclerotherapy and surgical stripping for great saphenous varicose veins. Br J Surg 2011;98: 1079-87. DOI: 10.1002/bjs. 7555 .

17. Figueiredo M, de Araujo SP, Figueiredo MF. Late follow-upof saphenofemoral junction ligation combined with ultrasound-guided foam sclerotherapy in patients with venous ulcers. Ann Vasc Surg. 2012 Oct;26(7):977-81. doi: 10.1016/j.avsg.2012.03.013.

18. Scurr JR, Fisher RK, Wallace SB, Gilling-Smith GL. Anaphylaxisfollowing foam sclerotherapy: A life threatening complication of non-invasive treatment for varicose veins. Eur J Vasc Endovasc Surg Extra 2007;13:87-9.

19. Brzoza Z, Kasperska-Zajac A, Rogala E, Rogala B. Anaphylactoid reaction after the use of sodium tetradecyl sulfate: a case report. Angiology. 2007 OctNov;58(5):644-6.

20. Guex JJ, Schliephake DE, Otto J, Mako S, Allaert FA. The French polidocanol study on long-term side effects: a survey covering 3,357 patient years. Dermatol Surg. 2010 Jun;36 Suppl 2:993-1003. doi: 10.1111/j.1524-4725.2009.01407.x.

21. Morrison N, Neuhardt DL, Rogers CR, McEown J, Morrison T, Johnson E, Salles-Cunha SX. Comparisons of side effects using air and carbon dioxide foam for endovenous chemical ablation. J Vasc Surg. 2008 
Apr;47(4):830-6. doi: 10.1016/j.jvs.2007.11.020. Epub 2008 Feb 1.

22. Blaise S, Bosson JL, Diamand JM. Ultrasoundguided sclerotherapy of the great saphenous vein with $1 \%$ vs. $3 \%$ polidocanol foam: a multicentre doubleblind randomised trial with 3-year follow-up. Eur J Vasc Endovasc Surg. 2010 Jun;39(6):779-86. doi: 10.1016/j.ejvs.2010.01.022. Epub 2010 Mar 4.

23. Lurie F, Creton D, Eklof B, Kabnick L, Kistner R, Pichot $\mathrm{O}$, et al. Prospective randomized study of endovenous radiofrequency obliteration (Closure procedure) versus ligation and stripping in a selected patient population (EVOLVeS Study). Journal of Vascular Surgery 2003;38(2):207-214.

24. Subramonia S, Lees T.Sensoryabnormalities and bruisingafterlongsaphenous veinstripping: impact on short-termquality of life. J Vasc Surg. 2005 Sep; 42(3):510-4.

25. Vasquez MA, Wang J, Mahathanaruk M, Buczkowski G, Sprehe E, Dosluoglu HH. The utility of the venous clinical severity score in 682 limbs treated by radiofrequency saphenous vein ablation. Journal of Vascular Surgery 2007;45(5):1008-1015.

26. Christenson JT, Gueddi S, Gemayel G, Bounameaux H. Prospective randomizedtrial comparingen dovenouslaser ablation and surgery for treatment of primary great saphenousvaricose veins with a 2-yearfollow-up.J Vasc Surg. 2010 Nov;52 (5):1234-41. doi: 10.1016/j.jvs.2010.06.104.

27. Evi Kalodiki, Christopher R Lattimer, Mustapha Azzam, Emad Shawish, Dimitris Bountouroglou, George Geroulakos. Long-term results of a randomized controlled trial on ultrasound-guided foam sclerotherapy combined with saphenofemoral ligation vs standard surgery for varicose veins. Journal of Vascular Surgery 2012;55(2):451-7.
28. Shadid N, Ceulen R, Nelemans P, C Dirksen, J Veraart, GW Schurink, et al. Randomized clinical trial of ultrasound-guided foam sclerotherapy versus surgery for the incompetent great saphenous vein. $\mathrm{Br} \mathrm{J}$ Surg. 2012 Aug;99(8):1062-70. doi: 10.1002/bjs.8781. Epub 2012 May 25.

29. Myers KA, Jolley D, Clough A, Kirwan J. Outcome of ultrasound-guided sclerotherapy for varicose veins: medium-term results assessed by ultrasound surveillance. Eur J Vasc Endovasc Surg. 2007 Jan;33(1):116-21. Epub 2006 Oct 24.

30. Gillet JL, Guedes JM, Guex JJ, Hamel-Desnos C, Schadeck M, Lausecker M, et al. Side-effects and complications of foam sclerotherapy of the great and small saphenous veins: A controlled multicentre prospective study including 1025 patients. Phlebology 2009;24(3):131-8.

31. Hamahata A, Yamaki T, Sakurai H. Outcomes of ultrasound-guided foam sclerotherapy for varicose veins of the lower extremities: a single center experience. Dermatol Surg. 2011 Jun;37(6):804-9. doi: 10.1111 /j.1524-4725.2011.02003..x.

32. Myers KA, Jolley D. Factors affecting the risk of deep venous occlusion after ultrasound-guided sclerotherapy for varicose veins. Eur J Vasc Endovasc Surg. 2008 Nov;36(5):602-5. doi: 10.1016 /j.ejvs.2008. 06.026. Epub 2008 Aug 20.

33. Hamel-Desnos C, Allaert FA. Liquid versus foam sclerotherapy. Phlebology. 2009 Dec; 24(6):240-6. doi: 10.1258/phleb.2009.009047.

34. Maurya AK, Singh S, Sachdeva V, Nath B, Verma SC, Gupta PK. Outcome of Ultrasound Guided Foam Sclerotherapy Treatment for Varicose Veins: Procedure is Standard and Need No Further Study. Indian J Vasc Endovasc Surg 2015;2:96-100.

\section{How to cite this article?}

Singh S, Maurya A.K, Singh M, Sachdeva V. Outcome of ultrasound guided foam sclerotherapy (UGFS) treatment for varicose veins: A four years retrospective study at a tertiary care hospital in north India. Int J Med Res Rev 2016;4(5):700-706.doi: 10.17511/ijmrr.2016.i05.06. 